Institute for Computational Mathematics

Hong Kong Baptist University

ICM Research Report

09-15 


\title{
High Order Compact Finite Difference Schemes For Helmholtz Equation With discontinuous coefficient
}

\author{
Xiufang Feng \\ School of Mathematics and Computer Sciences, Ningxia University, Yinchuan, China. \\ Email: xfeng@ncsu.edu \\ Zhilin Li \\ Center for Research in Scientific Computation \& Department of Mathematics, North Carolina State \\ University, Raleigh, NC 27695, USA. \\ Email: zhilin@ncsu.edu \\ Zhonghua Qiao \\ Institute for Computational Mathematics \& Department of Mathematics, Hong Kong Baptist \\ University, Kowloon, Hong Kong. \\ Email: zqiao@hkbu.edu.hk
}

\begin{abstract}
In this paper, third and fourth order compact finite difference schemes are proposed for solving Helmholtz equations with discontinuous wave numbers along straight interfaces in two space dimensions. To keep the compactness of the finite difference schemes and get global high order schemes, even at the interface where the wave number is discontinuous, the idea of the immerse interface method is employed. Numerical experiments are included to confirm the efficiency and accuracy of the proposed methods.
\end{abstract}

Mathematics subject classification: 65N06.

Key words: Helmholtz equation, compact finite difference scheme, discontinuous wave numbers, immerse interface method, nine-point stencil

\section{Introduction}

In this paper, we consider the following two-dimensional Helmholtz equation

$$
\Delta u+k_{0}^{2} \nu(x) u=f(x, y), \quad \text { or } \quad \Delta u+k^{2} u=f(x, y), \quad(x, y) \in \Omega
$$

in a rectangular domain $\Omega$ with a Dirichlet boundary condition, where $\Delta=\frac{\partial^{2}}{\partial x^{2}}+\frac{\partial^{2}}{\partial y^{2}}$ is the Laplace operator. The material coefficient $\nu(x)$ is assumed to be piecewise constant and has a finite jump across a straight line $\Gamma=\left\{(x, y), x=x_{0}\right\}$ in the domain, see [7] for a reference and applications of such a problem. For convenience, we will use the notation $k^{2}=k_{0}^{2} \nu(x)$ which is piecewise constant. Across the interface, the solution satisfies the following natural jump conditions

$$
[u]=0, \quad\left[u_{x}\right]=0, \quad\left[u_{y}\right]=0 .
$$

Notice that the second or higher order partial derivatives with respect to $x$, and the source term $f(x, y)$ may be discontinuous across the interface, see Fig.1.1 for an illustration.

Helmholtz equations describe many physical phenomena, including acoustic, elastic, and electromagnetic waves. Standard numerical methods, such as the boundary element method [16], finite element methods [12,19], and finite difference methods [25,26], have been employed 


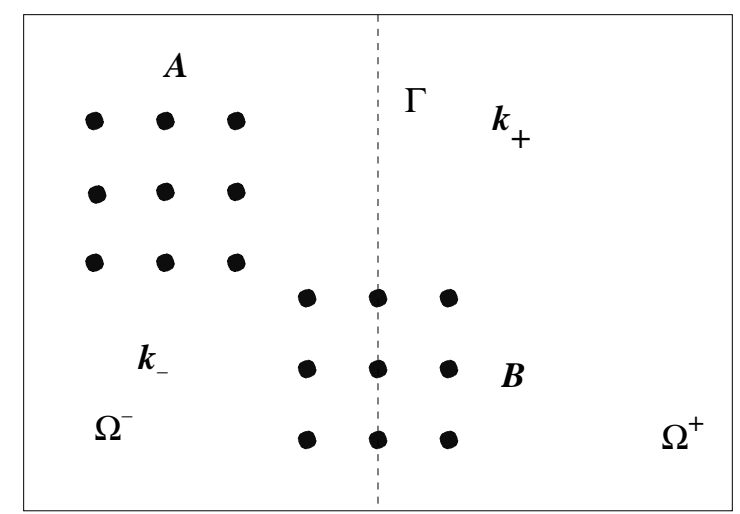

Fig. 1.1. A diagram of the problem and the finite difference stencil.

to solve the Helmholtz equation. Applications of the Helmholtz equations with discontinuous wave numbers can be found, for example, $[7,15]$.

The main difficulty in solving Helmholtz equations is that the solution is highly oscillatory for high wave number. The phase error (pollution) of the computed solution obtained with low order discretization is large unless fine meshes are used per wavelength, see for example [12]. A fine mesh would lead to a large system of equations which may be computationally prohibitive. Many different approaches have been proposed to reduce the phase error. For example, the highorder finite element method was proposed in [10]; the $h$-version and $h$-p-version finite element methods were proposed in $[13,14]$. In [19], a standard bilinear finite element together with a modified quadrature rule were used, which led to fourth-order phase accuracy on orthogonal uniform meshes for a constant wave number. Recently, high-order accurate methods, such as spectral methods and high order finite difference schemes, have been successfully developed in solving the Helmholtz equation when $k$ is constant; see e.g., $[2,3,17,18]$. But when $k$ is a piecewise constant, it becomes more difficult to get high order methods. In this case, the solution and its first order derivative are continuous everywhere [15], whereas the second order and higher derivatives or the right hand side $f(x, y)$ can have jumps at the discontinuity of $k$. In [6], Gustafsson and Wahlund analyzed the effect of discontinuous coefficients on the phase and amplitude errors. It was shown that the schemes in [5] were only first order accurate even if they are second or fourth order accurate for smooth solutions. In [7], Baruch et. al. constructed high order finite volume schemes for the one-dimensional Helmholtz equation with discontinuous coefficients based on the integral form of the Helmholtz equation. Their schemes can keep global higher order accuracy in the presence of discontinuities in the coefficients.

In this paper, we propose compact high-order (third and fourth) finite difference schemes to solve the two-dimensional Helmholtz equation with piecewise constant wave numbers. The compact high-order schemes are developed using the continuation of solutions by the Taylor series expansion and the immersed interface method, see for example, [23, 24]. Our high order finite difference schemes are based on the centered nine-point stencil, hence, the scheme is called compact, see [1] for the definition. The third order compact scheme developed in this paper is simple and easy to derive. The fourth order compact scheme may be necessary if 
the wave number is relatively large. Compact schemes have been developed for a variety of elliptic equations, see e.g., [8,9,11]. And it has also been successfully used to solve the Stokes equations in $[20,21]$. A compact fourth order finite difference method was proposed to solve the Helmholtz equation with a constant wave number $k$ in recent work [4]. One of advantages of compact schemes is that it can produce highly accurate numerical solutions without requiring extra boundary conditions. Furthermore, the compact scheme results in matrices that have much smaller band-width compared with non-compact schemes.

\subsection{The general form of the compact scheme}

Without loss of generality, we consider a square domain $\Omega=(a, b) \times(c, d)$. First we generate a mesh: $\left(x_{i}, y_{j}\right), x_{i}=a+i h_{x}, i=0,1, \ldots, M, y_{j}=c+j h_{y}, j=0,1, \ldots, N$, where $h_{x}=(b-a) / M$ and $h_{y}=(d-c) / M$ are the spacial mesh-sizes. To simplify the notation, we assume that $h_{x}=h_{y}=h$ and $M=N$. In general, the nine-point compact finite difference scheme can be written as

$$
\begin{aligned}
& a_{0} U_{i-1, j-1}+a_{1} U_{i-1, j}+a_{2} U_{i-1, j+1}+a_{3} U_{i, j-1}+a_{4} U_{i, j} \\
& \quad+a_{5} U_{i, j+1}+a_{6} U_{i+1, j-1}+a_{7} U_{i+1, j}+a_{8} U_{i+1, j+1}=F_{i j} .
\end{aligned}
$$

We use the upper case $U_{i j}$ to represent the solution of the finite difference equations while we use $u(x, y)$ to express the true solution to the Helmholtz equation, that is, $U_{i j} \approx u\left(x_{i}, y_{j}\right)$. If $k^{2}$ is continuous, as in the case for regular grid points where the interface $\Gamma$ does not cut through the centered nine-point stencil, the coefficients $a_{i}, i=0,1,2, \ldots, 8$ are obtained from the following finite difference equation.

$$
\begin{aligned}
\left(1+\frac{h^{2}}{12} \delta_{y}^{2}\right) \delta_{x}^{2} U_{i j}+\left(1+\frac{h^{2}}{12} \delta_{x}^{2}\right) \delta_{y}^{2} U_{i j} & +\left(1+\frac{h^{2}}{12} \delta_{x}^{2}+\frac{h^{2}}{12} \delta_{y}^{2}\right) k^{2} U_{i j} \\
& =\left(1+\frac{h^{2}}{12} \delta_{x}^{2}+\frac{h^{2}}{12} \delta_{y}^{2}\right) f_{i j}
\end{aligned}
$$

The central finite difference operator $\delta_{x}^{2}$ and $\delta_{y}^{2}$ are given by

$$
\delta_{x}^{2} U_{i j}=\frac{U_{i-1, j}-2 U_{i, j}+U_{i+1, j}}{h^{2}}, \quad \delta_{y}^{2} U_{i j}=\frac{U_{i, j-1}-2 U_{i, j}+U_{i, j+1}}{h^{2}} .
$$

By expanding the finite difference operator, we get the following finite difference coefficients:

$$
\begin{aligned}
& a_{0}=a_{2}=a_{6}=a_{8}=\frac{1}{6}, \quad a_{1}=a_{3}=a_{5}=a_{7}=\frac{h^{2} k^{2}}{12}+\frac{2}{3}, \\
& a_{4}=\frac{8 h^{2} k^{2}}{12}-\frac{10}{3} .
\end{aligned}
$$

The right hand side $F_{i, j}$ is given by

$$
F_{i, j}=\frac{h^{2}}{12}\left(f_{i+1, j}+f_{i-1, j}+f_{i, j+1} \cdot+f_{i, j-1}+8 f_{i, j}\right) .
$$


At irregular grid points where the nine-point stencil involves the discontinuity in $k^{2}$, see Fig. 1.1 stencil B for an illustration, we need to modify the finite difference coefficients and the right hand side to take into account of the discontinuity in $k^{2}$ and $f(x, y)$. Since the discontinuity is across an interface that is parallel to the $y$-axis, we set the interface as one of grid lines. As often done in numerical methods for interface problems, we set any point on the interface as in the domain of $\Omega^{-}$, that is, $\Gamma \subset \Omega^{-}$. The derivation of the third and fourth order compact schemes are given in the next two sections, followed by numerical examples. We assume a Dirichlet boundary condition for the Helmholtz equations.

\section{The third-order compact finite difference scheme}

In this section, we derive the third-order compact finite difference scheme for solving the Helmholtz equation. We use the fourth order compact finite difference scheme at regular grid points, that are all grid points except those on the interface. Irregular grid points are those on the interface $\Gamma$.

The finite difference coefficients of the third order compact scheme are given by

$$
\begin{aligned}
& a_{0}=a_{2}=\frac{1}{6}\left(1-\frac{h^{2}}{6}\left[k^{2}\right]\right), \quad a_{1}=\left(\frac{h^{2} k_{-}^{2}}{12}+\frac{2}{3}\right)\left(1-\frac{h^{2}}{6}\left[k^{2}\right]\right), \\
& a_{3}=a_{5}=\frac{h^{2} k_{-}^{2}}{12}+\frac{2}{3}+\frac{h^{2}}{9}\left[k^{2}\right], \\
& a_{4}=\frac{8 h^{2} k_{-}^{2}}{12}-\frac{10}{3}+\frac{2 h^{2}}{3}\left[k^{2}\right]\left(\frac{h^{2} k_{-}^{2}}{12}+\frac{2}{3}\right), \\
& a_{6}=a_{8}=\frac{1}{6}, \quad a_{7}=\frac{h^{2} k_{-}^{2}}{12}+\frac{2}{3}
\end{aligned}
$$

The right hand side $F_{i, j}$ is given by

$$
\begin{aligned}
F_{i, j}= & a_{6}\left(\frac{h^{2}}{2}[f]_{i, j+1}+\frac{h^{3}}{6}\left[f_{x}\right]_{i, j+1}\right)+a_{7}\left(\frac{h^{2}}{2}[f]_{i, j}+\frac{h^{3}}{6}\left[f_{x}\right]_{i, j}\right) \\
& +a_{6}\left(\frac{h^{2}}{2}[f]_{i, j-1}+\frac{h^{3}}{6}\left[f_{x}\right]_{i, j-1}\right) \\
& +\frac{h^{2}}{12}\left(f_{i+1, j}^{-}+f_{i-1, j}^{-}+f_{i, j+1}^{-}+f_{i, j-1}^{-}+8 f_{i, j}^{-}\right) .
\end{aligned}
$$

\subsection{Derivation of the third order compact finite difference scheme at irregular grid points}

Let $\left(x_{i}, y_{j}\right)$ be an irregular grid point. As noted before, the grid point is on the interface $\Gamma$ and belongs to the $\Omega^{-}$domain. Thus the grid points $\left(x_{i+1}, y_{l}\right), l=j-1, j, j+1$, are three grid points from the $\Omega^{+}$side. The idea in deriving the compact finite difference scheme is based on the continuation of the solution of $u(x, y)$ in the $\Omega^{-}$domain to the $\Omega^{+}$. Let us write the finite 
difference scheme as

$$
\begin{aligned}
& \frac{1}{6}\left(U_{i+1, j+1}+U_{i-1, j+1}+U_{i-1, j-1}+U_{i+1, j-1}\right) \\
& +\frac{2}{3}\left(U_{i+1, j}+U_{i-1, j}+U_{i, j+1}+U_{i, j-1}\right)-\frac{10}{3} U_{i, j} \\
& +\frac{k^{2} h^{2}}{12}\left(U_{i+1, j}+U_{i-1, j}+U_{i, j+1}+U_{i, j-1}+8 U_{i, j}\right) \\
& =\frac{h^{2}}{12}\left(f_{i+1, j}^{-}+f_{i-1, j}^{-}+f_{i, j+1}^{-}+f_{i, j-1}^{-}+8 f_{i, j}^{-}\right) \\
& \quad+C_{1}\left(U_{i-1, j-1}, \cdots, U_{i+1, j+1}\right) \\
& \quad+C_{2}\left(\left[k^{2}\right],[f]_{i, j-1}, \cdots,\left[f_{x}\right]_{i, j-1}\right)
\end{aligned}
$$

where the correction terms $C_{1}$ and $C_{2}$ are zero at regular grid points. Note that we should use the values of $f_{i+1, l}$ from the same side as $f_{i, l}, l=j-1, j, j+1$, that is, the $\Omega^{-}$side. If $f_{i+1, l}$ is not available, we can use a third or higher order extrapolation scheme to get it. We should choose $C_{1}$ and $C_{2}$ such that the local truncation error at the irregular grid point has the magnitude $O\left(h^{2}\right)$ or smaller. Note that, since the local truncation errors have order $O\left(h^{4}\right)$ at regular grid points, and the number of irregular grid points has order $O(N)$, the global accuracy will be of order $O\left(h^{3}\right)$, see, for example, [22,23] for the justification.

To derive the third order scheme, we need the following interface relations.

Lemma 2.1. Assume $f(x, y)$ is piecewise $C^{1}\left(\Omega^{ \pm}\right)$which leads to the fact that $u(x, y)$ is piecewise $C^{3}\left(\Omega^{ \pm}\right)$. We have the following jump conditions in addition to those in (1.2).

$$
\begin{array}{ll}
{\left[u_{y y}\right]=0, \quad\left[u_{x y}\right]=0, \quad\left[u_{x x}\right]=-\left[k^{2}\right] u^{-}+[f],} \\
{\left[u_{x x x}\right]=-\left[k^{2}\right] u_{x}^{-}+\left[f_{x}\right], \quad\left[u_{x x y}\right]=-\left[k^{2}\right] u_{y}^{-}+\left[f_{y}\right],} \\
{\left[u_{y y x}\right]=0, \quad\left[u_{y y y}\right]=0 .} &
\end{array}
$$

The proof is straightforward.

The local truncation error of the finite difference scheme (2.3) is

$$
\begin{aligned}
T_{i j}= & \frac{1}{6}\left(u\left(x_{i+1}, y_{j+1}\right)+u\left(x_{i-1}, y_{j+1}\right)+u\left(x_{i-1}, y_{j-1}\right)+u\left(x_{i+1}, y_{j-1}\right)\right) \\
& +\frac{2}{3}\left(u\left(x_{i+1}, y_{j}\right)+u\left(x_{i-1}, y_{j}\right)+u\left(x_{i}, y_{j+1}\right)+u\left(x_{i}, y_{j-1}\right)\right)-\frac{10}{3} u\left(x_{i}, y_{j}\right) \\
& +\frac{k^{2} h^{2}}{12}\left(u\left(x_{i+1}, y_{j}\right)+u\left(x_{i-1}, y_{j}\right)+u\left(x_{i}, y_{j+1}\right)+u\left(x_{i}, y_{j-1}\right)+8 u\left(x_{i}, y_{j}\right)\right) \\
& -\frac{h^{2}}{12}\left(f_{i+1, j}^{-}+f_{i-1, j}^{-}+f_{i, j+1}^{-}+f_{i, j-1}^{-}+8 f_{i, j}^{-}\right) \\
& -C_{1}\left(u\left(x_{i-1}, y_{j-1}\right), \cdots, u\left(x_{i+1}, y_{j+1}\right)\right) \\
- & C_{2}\left(\left[k^{2}\right],[f]_{i, j-1}, \cdots,\left[f_{x}\right]_{i, j-1}\right) .
\end{aligned}
$$


The idea of deriving the correction term is from the immersed interface method [22, 23]. We use the Taylor expansion and jump conditions to express the local truncation error in terms of the values of $u(x, y)$ from the - side, then we can determine the correction terms $C_{1}$ and $C_{2}$ by matching the differential equation up to third order partial derivatives with the finite difference scheme.

We use the Taylor expansion for the terms that are involved in the compact finite difference from the $\Omega^{+}$side. We have the following for those grid points that are in $\Omega^{+}$side:

$$
\begin{aligned}
u\left(x_{i+1}, y_{j}\right)= & u^{+}\left(x_{i+1}, y_{j}\right)=u^{+}\left(x_{i}, y_{j}\right)+h u_{x}^{+}\left(x_{i}, y_{j}\right) \\
& +\frac{h^{2}}{2} u_{x x}^{+}\left(x_{i}, y_{j}\right)+\frac{h^{3}}{6} u_{x x x}^{+}\left(x_{i}, y_{j}\right)+O\left(h^{4}\right), \\
u\left(x_{i+1}, y_{j+1}\right)= & u^{+}\left(x_{i+1}, y_{j+1}\right)=u^{+}\left(x_{i}, y_{j+1}\right)+h u_{x}^{+}\left(x_{i}, y_{j+1}\right) \\
& +\frac{h^{2}}{2} u_{x x}^{+}\left(x_{i}, y_{j+1}\right)+\frac{h^{3}}{6} u_{x x x}^{+}\left(x_{i}, y_{j+1}\right)+O\left(h^{4}\right), \\
u\left(x_{i+1}, y_{j-1}\right)= & u^{+}\left(x_{i+1}, y_{j-1}\right)=u^{+}\left(x_{i}, y_{j-1}\right)+h u_{x}^{+}\left(x_{i}, y_{j-1}\right) \\
& +\frac{h^{2}}{2} u_{x x}^{+}\left(x_{i}, y_{j-1}\right)+\frac{h^{3}}{6} u_{x x x}^{+}\left(x_{i}, y_{j-1}\right)+O\left(h^{4}\right) .
\end{aligned}
$$

We manipulate (2.6) to show the details by replacing the + terms in terms of those from side and the jump relations. The other two terms (2.7) and (2.8) are treated in the same way. We carry out the following derivation

$$
\begin{aligned}
u^{+}\left(x_{i+1}, y_{j}\right)= & u^{+}\left(x_{i}, y_{j}\right)+h u_{x}^{+}\left(x_{i}, y_{j}\right)+\frac{h^{2}}{2} u_{x x}^{+}\left(x_{i}, y_{j}\right)+\frac{h^{3}}{6} u_{x x x}^{+}\left(x_{i}, y_{j}\right)+O\left(h^{4}\right) \\
= & u^{-}\left(x_{i}, y_{j}\right)+h u_{x}^{-}\left(x_{i}, y_{j}\right)+\left.\frac{h^{2}}{2}\left(u_{x x}^{-}-\left[k^{2}\right] u^{-}+[f]\right)\right|_{\left(x_{i}, y_{j}\right)} \\
& +\left.\frac{h^{3}}{6}\left(u_{x x x}^{-}-\left[k^{2}\right] u_{x}^{-}+\left[f_{x}\right]\right)\right|_{\left(x_{i}, y_{j}\right)}+O\left(h^{4}\right) \\
= & u^{-}\left(x_{i+1}, y_{j}\right)-\left.\frac{h^{2}}{2}\left(\left[k^{2}\right] u^{-}-[f]\right)\right|_{\left(x_{i}, y_{j}\right)}-\left.\frac{h^{3}}{6}\left(\left[k^{2}\right] u_{x}^{-}-\left[f_{x}\right]\right)\right|_{\left(x_{i}, y_{j}\right)}+O\left(h^{4}\right),
\end{aligned}
$$

where $u^{-}\left(x_{i+1}, y_{j}\right)$ is the extension of $u(x, y)$ from the $\Omega^{-}$domain to the grid point $\left(x_{i+1}, y_{j}\right)$ via the Taylor expansion. We approximate $u_{x}^{-}\left(x_{i}, y_{j}\right)$ above using the backward finite difference formula

$$
u_{x}^{-}\left(x_{i}, y_{j}\right)=\frac{1}{h}\left(u^{-}\left(x_{i}, y_{j}\right)-u^{-}\left(x_{i-1}, y_{j}\right)\right)+O(h)
$$

Thus we have

$$
\begin{aligned}
u^{+}\left(x_{i+1}, y_{j}\right) \approx & u^{-}\left(x_{i+1}, y_{j}\right)-\left.\frac{h^{2}}{2}\left(\left[k^{2}\right] u^{-}-[f]\right)\right|_{\left(x_{i}, y_{j}\right)} \\
& -\frac{h^{3}}{6}\left(\frac{\left[k^{2}\right]}{h}\left(u^{-}\left(x_{i}, y_{j}\right)-u^{-}\left(x_{i-1}, y_{j}\right)\right)-\left.\left[f_{x}\right]\right|_{\left(x_{i}, y_{j}\right)}\right) .
\end{aligned}
$$

It is clear now that the part of $C_{1}$ should include

$$
-\frac{2 h^{2}}{3}\left[k^{2}\right] U_{i, j}+\frac{h^{2}}{6}\left[k^{2}\right] U_{i-1, j}
$$


while the part of $C_{2}$ should include

$$
\frac{h^{2}}{2}[f]_{i, j}+\frac{h^{3}}{6}\left[f_{x}\right]_{i, j} .
$$

The contributions to $C_{1}$ and $C_{2}$ from $u\left(x_{i+1}, y_{j+1}\right)$ and $u\left(x_{i+1}, y_{j-1}\right)$ can be derived in the exact the same way. In fact, we just need to change the subscript $j$ with $j+1$ or $j-1$ in the above expressions. Thus by collecting all the corresponding terms, we get $C_{1}$ and $C_{2}$ for the third order scheme

$$
\begin{aligned}
C_{1}=-\frac{2 h^{2}}{3} & {\left[k^{2}\right] U_{i, j-1}+\frac{h^{2}}{6}\left[k^{2}\right] U_{i-1, j-1}-\frac{2 h^{2}}{3}\left[k^{2}\right] U_{i, j}+\frac{h^{2}}{6}\left[k^{2}\right] U_{i-1, j} } \\
& -\frac{2 h^{2}}{3}\left[k^{2}\right] U_{i, j+1}+\frac{h^{2}}{6}\left[k^{2}\right] U_{i-1, j+1}, \\
C_{2}= & \frac{1}{6}\left(\frac{h^{2}}{2}[f]_{i, j+1}+\frac{h^{3}}{6}\left[f_{x}\right]_{i, j+1}\right)+\left(\frac{h^{2}}{12} k_{-}^{2}+\frac{2}{3}\right)\left(\frac{h^{2}}{2}[f]_{i, j}+\frac{h^{3}}{6}\left[f_{x}\right]_{i, j}\right) \\
+ & \frac{1}{6}\left(\frac{h^{2}}{2}[f]_{i, j-1}+\frac{h^{3}}{6}\left[f_{x}\right]_{i, j-1}\right) .
\end{aligned}
$$

Combining $C_{1}$ and $C_{2}$ with the standard 9-point compact scheme, we get the finite difference coefficients (2.1)-(2.2) for the third order compact scheme.

Remark 2.1. The local truncation errors of the third order compact scheme have order $O\left(h^{4}\right)$ at regular grid points, and $O\left(h^{2}\right)$ at irregular grid points. This is because we expand the Taylor expansion up to all third order partial derivatives and the finite difference coefficients have the magnitude of $1 / h^{2}$. Since the interface $\Gamma$ is one dimensional, the global error has order $O\left(h^{3}\right)$.

\section{The fourth order compact scheme}

The third order compact scheme is relative easy to derive and implement. That is one of the most important advantage of the method. We just need to expand $u\left(x_{i+1}, y_{l}\right)$ terms, $l=j-1$, $j, j+1$, at $\left(x_{i}, y_{l}\right)$. The correction term that contains $h^{3} u_{x}^{-}$is approximated using the function values within the nine-point stencil. However, the third order compact scheme does not match the fourth order scheme at regular grid points. In this section, we derive the fourth order compact scheme. The computational cost of the fourth order scheme is about the same as that of the third order one. The correction terms will involve more terms that are related to fourth order partial derivatives.

The finite difference coefficients of the fourth order compact scheme are given by 


$$
\begin{aligned}
& a_{0}=a_{2}=a_{6}=a_{8}=\frac{1}{6}, \\
& a_{1}=\beta\left(1-\frac{\alpha}{1-\alpha}\right)-\frac{\alpha}{3}\left(1+\frac{\alpha}{1-\alpha}\right), \\
& a_{3}=a_{5}=\beta\left(1-\frac{\alpha}{1-\alpha}\right)+\frac{2 \alpha}{3}\left(1-\frac{\alpha}{2(1-\alpha)}\right), \\
& a_{4}=-\frac{26}{3}+8 \beta+\frac{2 \alpha}{3}-\frac{\gamma}{3}-\frac{1}{1-\alpha}\left(\beta+\frac{\alpha}{3}\right)(-8 \alpha+\gamma), \\
& a_{7}=\frac{1}{1-\alpha}\left(\beta+\frac{\alpha}{3}\right)
\end{aligned}
$$

and

$$
\begin{aligned}
& F_{i j}=\frac{1}{6} R_{1}+\frac{1}{1-\alpha}\left(\beta+\frac{\alpha}{3}\right) R_{2}+\frac{1}{6} R_{3} \\
& +\frac{h^{2}}{12}\left(f_{i+1, j}^{-}+f_{i-1, j}^{-}+f_{i, j+1}^{-}+f_{i, j-1}^{-}+8 f_{i, j}^{-}\right),
\end{aligned}
$$

where

$$
\begin{gathered}
\alpha=\frac{h^{2}}{12}\left[k^{2}\right], \quad \beta=\frac{2}{3}+\frac{h^{2}}{12} k_{-}^{2}, \quad \gamma=\frac{h^{4}}{24}\left[k^{4}\right], \\
R_{1}=\left(\frac{h^{2}}{2}[f]+\frac{h^{3}}{6}\left[f_{x}\right]+\frac{h^{3}}{2}\left[f_{y}\right]-\frac{h^{4}}{24}\left[k^{2} f\right]+\frac{5 h^{4}}{24}[f]_{y y}\right. \\
\left.\quad+\frac{h^{4}}{24}\left[f_{x x}\right]+\frac{h^{4}}{6}\left[f_{x y}\right]\right)\left.\right|_{\left(x_{i}, y_{j}\right)}, \\
R_{2}=\left.\left(\frac{h^{2}}{2}[f]+\frac{h^{3}}{6}\left[f_{x}\right]-\frac{h^{4}}{24}\left[k^{2} f\right]-\frac{h^{4}}{24}[f]_{y y}+\frac{h^{4}}{24}\left[f_{x x}\right]\right)\right|_{\left(x_{i}, y_{j}\right)} \\
R_{3}=\left(\frac{h^{2}}{2}[f]+\frac{h^{3}}{6}\left[f_{x}\right]-\frac{h^{3}}{2}\left[f_{y}\right]-\frac{h^{4}}{24}\left[k^{2} f\right]\right. \\
\left.\quad+\frac{5 h^{4}}{24}[f]_{y y}+\frac{h^{4}}{24}\left[f_{x x}\right]-\frac{h^{4}}{6}\left[f_{x y}\right]\right)\left.\right|_{\left(x_{i}, y_{j}\right)} .
\end{gathered}
$$

\subsection{Derivation of the fourth order compact finite difference scheme at irregular grid points}

The derivation process is similar to that of the third order compact finite difference scheme. So we will use the same correction notations $C_{1}$ and $C_{2}$. To get the fourth order scheme, we need to match the differential equation up to fourth order partial derivatives. Thus we need the following lemma in addition to Lemma 2.1. 
Lemma 3.1. Assume $f(x, y)$ is piecewise $C^{2}\left(\Omega^{ \pm}\right)$which leads to the fact that $u(x, y)$ is piecewise $C^{4}\left(\Omega^{ \pm}\right)$. We have the following jump conditions in addition to those in (1.2) and (2.4).

$$
\begin{aligned}
& {\left[u_{y y y x}\right]=0, \quad\left[u_{y y y y}\right]=0, \quad\left[u_{x x x y}\right]=-\left[k^{2}\right] u_{x y}^{-}+\left[f_{x y}\right],} \\
& {\left[u_{x x y y}\right]=-\left[k^{2}\right] u_{y y}^{-}+\left[f_{y y}\right],} \\
& {\left[u_{x x x x}\right]=2\left[k^{2}\right] u_{y y}^{-}+\left[k^{4}\right] u^{-}-\left[k^{2} f\right]+\left[f_{x x}\right]-\left[f_{y y}\right],}
\end{aligned}
$$

Proof: We show the proof for the last two expressions. The proof for others is straightforward. Differentiating the original differential equation twice with respect to $y$ and then taking the jump, we get

$$
\left[u_{x x y y}\right]+\left[u_{y y y y}\right]+\left[k^{2} u_{y y}\right]=\left[f_{y y}\right] .
$$

Since $u$ is continuous with respect to $y$, we get

$$
\left[u_{x x y y}\right]=-\left[k^{2}\right] u_{y y}^{-}+\left[f_{y y}\right] .
$$

Note that $u_{y y}$ is continuous, so we can write $u_{y y}=u_{y y}^{-}$. Differentiating the original equation twice with respect to $x$ and then taking the jump, we get

$$
\left[u_{x x x x}\right]+\left[u_{y y x x}\right]+\left[k^{2} u_{x x}\right]=\left[f_{x x}\right] .
$$

Multiplying $k^{2}$ to the original differential equation and then taking the jump, we have

$$
\left[k^{2} u_{x x}\right]=-\left[k^{2}\right] u_{y y}^{-}-\left[k^{4}\right] u^{-}+\left[k^{2} f\right] .
$$

Thus using $\left[u_{x x y y}\right]$ and $\left[k^{2} u_{x x}\right]$, we get

$$
\begin{aligned}
{\left[u_{x x x x}\right] } & =-\left[u_{y y x x}\right]-\left[k^{2} u_{x x}\right]+\left[f_{x x}\right] \\
& =\left[k^{2}\right] u_{y y}^{-}-\left[f_{y y}\right]+\left[k^{2}\right] u_{y y}^{-}+\left[k^{4}\right] u^{-}-\left[k^{2} f\right]+\left[f_{x x}\right] \\
& =2\left[k^{2}\right] u_{y y}^{-}+\left[k^{4}\right] u^{-}-\left[k^{2} f\right]+\left[f_{x x}\right]-[f]_{y y} .
\end{aligned}
$$

This completes the proof.

To derive the fourth order compact scheme at irregular grid points on the interface, again we use the continuation via the Taylor expansion. Let $\left(x_{i}, y_{j}\right)$ be such a grid point, we discuss how to choose $C_{1}$ and $C_{2}$ to minimize the local truncation error in (2.5). From the Taylor expansion, we have

$$
\begin{aligned}
u\left(x_{i+1}, y_{j+1}\right)= & u^{+}+h\left(u_{x}^{+}+u_{y}^{+}\right)+\frac{h^{2}}{2}\left(u_{x x}^{+}+2 u_{x y}^{+}+u_{y y}^{+}\right) \\
& +\frac{h^{3}}{6}\left(u_{x x x}^{+}+3 u_{x x y}^{+}+3 u_{x y y}^{+}+u_{y y y}^{+}\right) \\
& +\frac{h^{4}}{24}\left(u_{x x x x}^{+}+4 u_{x x x y}^{+}+6 u_{x x y y}^{+}+4 u_{x y y y}^{+}+u_{y y y}^{+}\right)+O\left(h^{5}\right),
\end{aligned}
$$


where all the quantities are evaluated at $\left(x_{i}, y_{j}\right)$. Note that now we expand $u\left(x_{i+1}, y_{j+1}\right)$ at $\left(x_{i}, y_{j}\right)$, but not at $\left(x_{i}, y_{j+1}\right)$ as we did in the third order compact scheme. The reason is that we need to keep the correction terms that involves $U_{l k}$ within the nine-point stencil. We expand $u\left(x_{i+1}, y_{j+1}\right)$ up to all the fourth order partial derivatives to get fourth order accuracy.

With the jump conditions from (1.2), (2.4), and (3.7), we replace the values of that have + superscript in terms of those from - side, we get

$$
\begin{aligned}
u\left(x_{i+1}, y_{j+1}\right)= & u^{-}+h\left(u_{x}^{-}+u_{y}^{-}\right)+\frac{h^{2}}{2}\left(u_{x x}^{-}-\left[k^{2}\right] u^{-}+[f]+2 u_{x y}^{-}+u_{y y}^{-}\right) \\
& +\frac{h^{3}}{6}\left(u_{x x x}^{-}-\left[k^{2}\right] u_{x}^{-}+\left[f_{x}\right]+3 u_{x x y}^{-}-3\left[k^{2}\right] u_{y}^{-}+3\left[f_{y}\right]+3 u_{x y y}^{-}+u_{y y y}^{-}\right) \\
& +\frac{h^{4}}{24}\left(u_{x x x x}^{-}+2\left[k^{2}\right] u_{y y}^{-}+\left[k^{4}\right] u^{-}-\left[k^{2} f\right]-[f]_{y y}+\left[f_{x x}\right]+4 u_{x x x y}^{-}\right. \\
& \left.-4\left[k^{2}\right] u_{x y}^{-}+4\left[f_{x y}\right]+6 u_{x x y y}^{-}-6\left[k^{2}\right] u_{y y}^{-}+6[f]_{y y}+4 u_{x y y y}^{-}+u_{y y y y}^{-}\right)+O\left(h^{5}\right) .
\end{aligned}
$$

To get a fourth order scheme, we need to use finite difference formulas to approximate $u_{x}^{-}$and $u_{y}^{-}$to second order; $u_{x y}^{-}$and $u_{y y}^{-}$to first order at the grid point $\left(x_{i}, y_{j}\right)$ to ensure that the local truncation errors at these grid points are of order $O\left(h^{3}\right)$. The finite difference approximations have to use the nine-point stencil to keep the finite difference compact. The finite difference approximation for $u_{x}^{-}, u_{y}^{-}, u_{x y}^{-}$, and $u_{y y}^{-}$are the following:

$$
\begin{aligned}
& u_{x}^{-}\left(x_{i}, y_{j}\right) \approx \frac{1}{2 h}\left(u^{-}\left(x_{i+1}, y_{j}\right)-u^{-}\left(x_{i-1}, y_{j}\right)\right), \\
& u_{y}^{-}\left(x_{i}, y_{j}\right) \approx \frac{1}{2 h}\left(u^{-}\left(x_{i}, y_{j+1}\right)-u^{-}\left(x_{i}, y_{j-1}\right)\right), \\
& u_{x y}^{-}\left(x_{i}, y_{j}\right) \approx \frac{1}{2 h^{2}}\left(u^{-}\left(x_{i}, y_{j+1}\right)-u^{-}\left(x_{i}, y_{j-1}\right)-u^{-}\left(x_{i-1}, y_{j+1}\right)+u^{-}\left(x_{i-1}, y_{j-1}\right)\right), \\
& u_{y y}^{-}\left(x_{i}, y_{j}\right) \approx \frac{1}{h^{2}}\left(u^{-}\left(x_{i}, y_{j+1}\right)-2 u^{-}\left(x_{i}, y_{j}\right)+u^{-}\left(x_{i}, y_{j-1}\right)\right) .
\end{aligned}
$$

We can see that all the grid points are still within the nine-point stencil. With such approximations, we can rewrite

$$
\begin{aligned}
u^{+}\left(x_{i+1}, y_{j+1}\right)= & u^{-}\left(x_{i+1}, y_{j+1}\right)+\left(-\frac{h^{2}}{6}\left[k^{2}\right]+\frac{h^{4}\left[k^{4}\right]}{24}\right) u^{-}\left(x_{i}, y_{j}\right)+\frac{h^{2}}{12}\left[k^{2}\right] u^{-}\left(x_{i-1}, y_{j}\right) \\
& +\frac{h^{2}}{6}\left[k^{2}\right] u^{-}\left(x_{i}, y_{j-1}\right)-\frac{h^{2}\left[k^{2}\right]}{2} u^{-}\left(x_{i}, y_{j+1}\right)+\frac{h^{2}}{12}\left[k^{2}\right] u^{-}\left(x_{i-1}, y_{j+1}\right) \\
& -\frac{h^{2}}{12}\left[k^{2}\right] u^{-}\left(x_{i-1}, y_{j-1}\right)-\frac{h^{2}}{12}\left[k^{2}\right] u^{-}\left(x_{i+1}, y_{j}\right)+R_{1}+O\left(h^{5}\right)
\end{aligned}
$$

where $R_{1}$ is given in (3.4), and $u^{-}\left(x_{i+1}, y_{j+1}\right)$ is the fifth order smooth extension of $u^{-}(x, y)$ to the point $\left(x_{i+1}, y_{j+1}\right)$. 
Thus we get the contribution to $C_{1}$ from $u\left(x_{i+1}, y_{j+1}\right)$ :

$$
\begin{aligned}
& \left(-\frac{h^{2}}{6}\left[k^{2}\right]+\frac{h^{4}\left[k^{4}\right]}{24}\right) U_{i, j}+\frac{h^{2}}{12}\left[k^{2}\right] U_{i-1, j}+\frac{h^{2}}{6}\left[k^{2}\right] U_{i, j-1} \\
& \quad-\frac{h^{2}\left[k^{2}\right]}{2} U_{i, j+1}+\frac{h^{2}}{12}\left[k^{2}\right] U_{i-1, j+1}-\frac{h^{2}}{12}\left[k^{2}\right] U_{i-1, j-1}-\frac{h^{2}}{12}\left[k^{2}\right] U_{i+1, j},
\end{aligned}
$$

and the contribution to $C_{2}$ is simply $R_{1}$.

Use almost the same procedure, we can get the contribution to $C_{1}$ and $C_{2}$ from $u\left(x_{i+1}, y_{j}\right)$ and $u\left(x_{i+1}, y_{j-1}\right)$. Thus by collecting all the corresponding terms, we get $C_{1}$ and $C_{2}$ for the fourth order scheme

$$
\begin{aligned}
& C_{1}=\left(-\frac{h^{2}}{6}\left[k^{2}\right]+\frac{h^{4}}{24}\left[k^{4}\right]\right) U_{i, j}+\frac{h^{2}}{12}\left[k^{2}\right] U_{i-1, j}+\frac{h^{2}}{6}\left[k^{2}\right] U_{i, j-1}-\frac{h^{2}}{2}\left[k^{2}\right] U_{i, j+1} \\
&+\frac{h^{2}}{12}\left[k^{2}\right] U_{i-1, j+1}-\frac{h^{2}}{12}\left[k^{2}\right] U_{i-1, j-1}-\frac{h^{2}}{12}\left[k^{2}\right] U_{i+1, j}-\frac{h^{2}}{12}\left[k^{2}\right] U_{i+1, j} \\
&+\left(-\frac{2 h^{2}}{3}\left[k^{2}\right]+\frac{h^{4}}{24}\left[k^{4}\right]\right) U_{i, j}+\frac{h^{2}}{12}\left[k^{2}\right] U_{i-1, j}+\frac{h^{2}}{12}\left[k^{2}\right] U_{i, j+1}+\frac{h^{2}}{12}\left[k^{2}\right] U_{i, j-1}, \\
&+\left(-\frac{h^{2}}{6}\left[k^{2}\right]+\frac{h^{4}}{24}\left[k^{4}\right]\right) U_{i, j}+\frac{h^{2}}{12}\left[k^{2}\right] U_{i-1, j}+\frac{h^{2}}{6}\left[k^{2}\right] U_{i, j+1} \\
& \quad-\frac{h^{2}}{2}\left[k^{2}\right] U_{i, j-1}-\frac{h^{2}}{12}\left[k^{2}\right] U_{i-1, j+1}+\frac{h^{2}}{12}\left[k^{2}\right] U_{i-1, j-1}-\frac{h^{2}}{12}\left[k^{2}\right] U_{i+1, j}, \\
& C_{2}=R_{1}+ R_{2}+R_{3},
\end{aligned}
$$

where $R_{1}, R_{2}$, and $R_{3}$ are given by (3.4)-(3.6). Each terms corresponds to the correction to the right-hand side from the three irregular grid points in the finite difference stencil. Combining $C_{1}$ and $C_{2}$ with the standard 9-point compact scheme, we get the finite difference coefficients (3.1)-(3.2) for the fourth order compact scheme.

Remark 3.1. The local truncation errors of the fourth order compact scheme have order $O\left(h^{4}\right)$ at regular grid points, and $O\left(h^{3}\right)$ at irregular grid points. This is because we expand the Taylor expansion up to all fourth order partial derivatives and the finite difference coefficients have the magnitude of $1 / h^{2}$ when we derive the finite difference scheme at irregular grid points. Since the interface $\Gamma$ is one dimensional, the global error has order $O\left(h^{4}\right)$.

Remark 3.2. If $k$ is a continuous constant and $f(x, y) \in C^{2}(\Omega)$, then the coefficients in (3.1)(3.6) are simply those of the standard fourth order compact scheme (1.3), (1.6)-(1.7).

\section{Numerical experiments}

In this section, we present two numerical examples that we have the exact solution to show the convergence of our third and fourth order compact schemes for solving the Helmholtz equation with a straight interface $x=x_{0}$. All computations are done using a Dell Desktop or a 
notebook computer. Most of computations are done within seconds or a few minutes depending the mesh size. A Dirichlet boundary condition is used. The error is measured in the $L_{\infty}$ norm for all the grid points and the convergence order is estimated using $r=\log \left(e_{h_{1}} / e_{h_{2}}\right) / \log \left(h_{1} / h_{2}\right)$ as a common practice in the literature.

\section{Example 4.1.}

In this example, we use the exact solution $u=\sin (\pi x) \sin (\pi y)$ to derive $f(x, y)$. The wave number $k$ has a finite jump across $x=1 / 2$, so does $f(x, y)$ within the domain $\Omega=(0,1) \times(0,1)$ which includes two parts $\Omega^{-}=\left(0, \frac{1}{2}\right] \times(0,1)$ and $\Omega^{+}=\left(\frac{1}{2}, 1\right) \times(0,1)$. The source term $f$ is given by

$$
f(x, y)= \begin{cases}-2 \pi^{2}+k_{-}^{2} \sin \pi x \sin \pi y & (x, y), \in \Omega^{-} \\ -2 \pi^{2}+k_{+}^{2} \sin \pi x \sin \pi y & (x, y), \in \Omega^{+}\end{cases}
$$

In Table 4.1, we show a grid refinement analysis with different wave numbers. In the secondthird column, we show the results with relatively small wave number $k_{-}^{2}=1$ and $k_{+}^{2}=9$; in the fourth-fifth column, we show the results with medium size wave number $k_{-}^{2}=25$ and $k_{+}^{2}=100$. In both cases, third order convergence can be clearly observed. In Table 4.2, we present the results obtained from the fourth order compact scheme. Fourth order convergence is clearly confirmed and the error is one order smaller than that of the third order one.

Table 4.1: A grid refinement analysis for Example 4.1 using the third order compact scheme with different wave numbers. Third order convergence is confirmed.

\begin{tabular}{|c|cc|cc|}
\hline & \multicolumn{2}{|c|}{$k_{-}^{2}=1, k_{+}^{2}=9$} & \multicolumn{2}{c|}{$k_{-}^{2}=25, k_{+}^{2}=100$} \\
\hline$N$ & error & order & error & order \\
\hline 16 & $2.8842 \mathrm{e}-4$ & & 0.0086 & \\
\hline 32 & $3.6520 \mathrm{e}-5$ & 2.9814 & 0.0011 & 2.9668 \\
\hline 64 & $4.5905 \mathrm{e}-6$ & 2.9920 & $1.3662 \mathrm{e}-4$ & 3.0093 \\
\hline 128 & $5.7529 \mathrm{e}-7$ & 2.9963 & $1.7050 \mathrm{e}-5$ & 3.0023 \\
\hline 256 & $7.1999 \mathrm{e}-8$ & 2.9982 & $2.1303 \mathrm{e}-6$ & 3.0006 \\
\hline 512 & $8.9994 \mathrm{e}-9$ & 3.0001 & $2.6625 \mathrm{e}-7$ & 3.0002 \\
\hline
\end{tabular}

In Table 4.3, we compare the third and fourth order compact scheme for the large wave number $k_{+}^{2}=992.25$. We choose this number so that the wave number is far from one of the eigenvalues in which the coefficient matrix is singular. Both schemes work well even with such a large jump of the wave number.

Note that in Table 4.2 and Table 4.3, the convergence rate for the fourth order scheme is deteriorated when $N=512$. This is because of the round-off errors have spoiled the convergence order when $h$ is too small. It is easy to show that there is a critical step size $h_{c} \sim C \epsilon^{1 /(p+2)}$ below which the run-off error will become dominate, where $p$ is convergence order of the global 
Table 4.2: A grid refinement analysis for Example 4.1 using the fourth order compact scheme with different wave numbers. Fourth order convergence is confirmed.

\begin{tabular}{|c|cc|cc|}
\hline & \multicolumn{2}{|c|}{$k_{-}^{2}=1, k_{+}^{2}=9$} & \multicolumn{2}{c|}{$k_{-}^{2}=25, k_{+}^{2}=100$} \\
\hline$N$ & error & order & error & order \\
\hline 16 & $5.7294 \mathrm{e}-6$ & & $1.3793 \mathrm{e}-5$ & \\
\hline 32 & $3.5852 \mathrm{e}-7$ & 3.9983 & $8.1485 \mathrm{e}-7$ & 4.0813 \\
\hline 64 & $2.2416 \mathrm{e}-8$ & 3.9995 & $5.0579 \mathrm{e}-8$ & 4.0099 \\
\hline 128 & $1.4020 \mathrm{e}-9$ & 3.9990 & $3.1595 \mathrm{e}-9$ & 4.0008 \\
\hline 256 & $8.9138 \mathrm{e}-11$ & 3.9753 & $2.0095 \mathrm{e}-10$ & 3.9748 \\
\hline 512 & $1.1538 \mathrm{e}-11$ & 2.9496 & $2.6062 \mathrm{e}-11$ & 2.9468 \\
\hline
\end{tabular}

Table 4.3: A comparison of the third and fourth order compact schemes for Example 4.1 with $k_{-}^{2}=1$, $k_{+}^{2}=992.25$.

\begin{tabular}{|c|cc|cc|}
\hline & third-order scheme & \multicolumn{2}{|c|}{ fourth-order scheme } \\
\hline$N$ & error & order & error & order \\
\hline 32 & $9.3486 \mathrm{e}-4$ & & $9.3545 \mathrm{e}-8$ & \\
\hline 64 & $1.0368 \mathrm{e}-4$ & 3.1726 & $5.3675 \mathrm{e}-9$ & 4.1233 \\
\hline 128 & $1.2581 \mathrm{e}-5$ & 3.0428 & $3.3586 \mathrm{e}-10$ & 3.9983 \\
\hline 256 & $1.5610 \mathrm{e}-6$ & 3.0107 & $2.1354 \mathrm{e}-11$ & 3.9753 \\
\hline 512 & $1.9840 \mathrm{e}-7$ & 3.0024 & $2.7633 \mathrm{e}-12$ & 2.9500 \\
\hline
\end{tabular}

error, $C$ is a constant and $\epsilon$ is the machine precision. For the third and fourth order compact schemes, $p=3$ or $p=4$. In our numerical tests, we use double precision, and hence $\epsilon=10^{-16}$. The critical step size $h_{c} \sim 6.3 C \times 10^{-4}$ for the third order scheme, and $h_{c} \sim 2.2 C \times 10^{-3}$ for the fourth order scheme. Note that $1 / N=1 / 512=0.002<2.2 \times 10^{-3}$. Thus the round-off errors will become dominant sooner as we increase the mesh size for higher order schemes, particularly for the fourth order one.

\section{Example 4.2.}

We consider the following problem: the interface is the line $x=\pi / 2$ within the domain $\Omega=$ $(0, \pi) \times(0, \pi)$ which includes two parts $\Omega^{-}=(0, \pi / 2] \times(0, \pi)$ and $\Omega^{+}=(\pi / 2, \pi) \times(0, \pi)$, the source item $f(x, y)$ is given as

$$
f(x, y)= \begin{cases}\left(2+\left(k_{-}^{2}-2\right)(x-\pi / 2)^{2}\right) \sin x \sin y+4(x-\pi / 2) \cos x \sin y, & (x, y) \in \Omega^{-}, \\ \left(2+\left(k_{+}^{2}-2\right)(x-\pi / 2)^{2}\right) \cos x \cos y-4(x-\pi / 2) \sin x \cos y, & (x, y) \in \Omega^{+} .\end{cases}
$$

The exact solution of the problem is

$$
u(x, y)= \begin{cases}\left(x-\frac{\pi}{2}\right)^{2} \sin x \sin y, & (x, y) \in \Omega^{-}, \\ \left(x-\frac{\pi}{2}\right)^{2} \cos x \cos y, & (x, y) \in \Omega^{+} .\end{cases}
$$


Unlike Example 4.1, the second order partial derivative $u_{x x}$ is discontinuous at the interface $x=\pi / 2$ in this example. In Table 4.4, we show a grid refinement analysis with different wave numbers. In the second-third column, we show the results with relatively small wave number $k_{-}^{2}=1$ and $k_{+}^{2}=9$; in the fourth-fifth column, we show the results with medium size wave number $k_{-}^{2}=25$ and $k_{+}^{2}=100$. In both cases, third order convergence can be clearly seen. In Table 4.5, we present the results obtained from the fourth order compact scheme. Fourth order convergence is clearly confirmed and the error is one order smaller than that of the third order one.

Table 4.4: A grid refinement analysis for Example 4.2 using the third order compact scheme with different wave numbers. Third order convergence is confirmed.

\begin{tabular}{|c|cc|cc|}
\hline & \multicolumn{2}{|c|}{$k_{-}^{2}=1, k_{+}^{2}=9$} & & \multicolumn{2}{|c|}{$k_{-}^{2}=25, k_{+}^{2}=100$} & \\
\hline$N$ & error & order & error & order \\
\hline 16 & 0.0048 & & 0.0386 & \\
\hline 32 & $5.8065 \mathrm{e}-4$ & 3.0473 & 0.0013 & 4.8920 \\
\hline 64 & $7.2289 \mathrm{e}-5$ & 3.0058 & $1.4950 \mathrm{e}-4$ & 3.1203 \\
\hline 128 & $9.0227 \mathrm{e}-6$ & 3.0021 & $1.8172 \mathrm{e}-5$ & 3.0404 \\
\hline 256 & $1.1274 \mathrm{e}-6$ & 3.0006 & $2.2552 \mathrm{e}-6$ & 3.0104 \\
\hline 512 & $1.4088 \mathrm{e}-7$ & 3.0005 & $2.8139 \mathrm{e}-7$ & 3.0026 \\
\hline
\end{tabular}

Table 4.5: A grid refinement analysis for Example 4.2 using the fourth order compact scheme with different wave numbers. Fourth order convergence is confirmed.

\begin{tabular}{|c|cc|cc|}
\hline & $k_{-}^{2}=1, k_{+}^{2}=9$ & & $k_{-}^{2}=25, k_{+}^{2}=100$ & \\
\hline$N$ & error & order & error & order \\
\hline 16 & $1.9976 \mathrm{e}-4$ & & $1.2797 \mathrm{e}-4$ & \\
\hline 32 & $1.1792 \mathrm{e}-5$ & 4.0824 & $7.5419 \mathrm{e}-6$ & 4.0847 \\
\hline 64 & $7.1269 \mathrm{e}-7$ & 4.0484 & $4.7952 \mathrm{e}-7$ & 3.9753 \\
\hline 128 & $4.3801 \mathrm{e}-8$ & 4.0242 & $2.9489 \mathrm{e}-8$ & 4.0233 \\
\hline 256 & $2.7156 \mathrm{e}-9$ & 4.0116 & $1.8327 \mathrm{e}-9$ & 4.0081 \\
\hline 512 & $1.6925 \mathrm{e}-10$ & 4.0040 & $1.1449 \mathrm{e}-10$ & 4.0007 \\
\hline
\end{tabular}

In figure 4.1 and figure 4.2, we show the error plot of the computed solutions using the third and fourth order compact schemes, respectively.

In Table 4.6 and Table 4.7, we compare the third and fourth order compact scheme for different wave number $k_{-}^{2}=1$ and $k_{+}^{2}=992.25$ in Table 4.6 as we did for Example 4.1; and $k_{-}^{2}=25$ and $k_{+}^{2}=90000$ in Table 4.7. We observe similar results as those for Example 4.1 that we have clear cut third order and fourth order convergence. For the large wave number case $k_{+}^{2}=90000$, either we need to use high order accurate schemes or very fine mesh to resolve the solution. In Table 4.7, we see that convergence rate of the third order compact scheme 


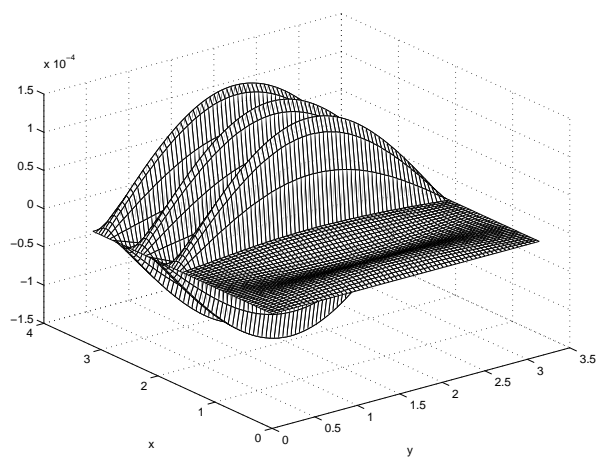

Fig. 4.1. The error plot for the numerical solution of Example 4.2 using the third order compact scheme with $k_{-}^{2}=25, k_{+}^{2}=100$, and $N=64$.

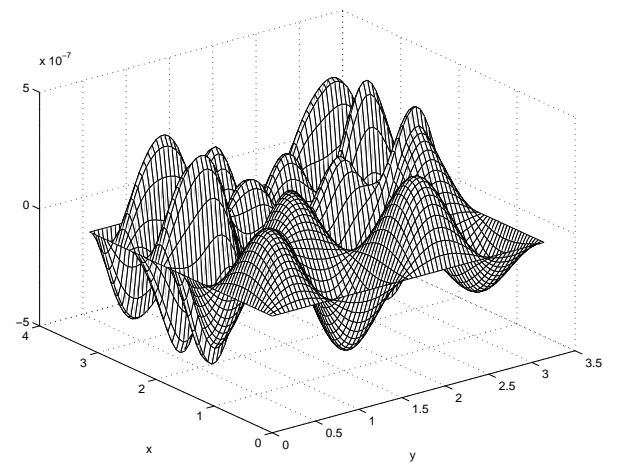

Fig. 4.2. The error plot for the numerical solution of Example 4.2 using the fourth order compact scheme with $k_{-}^{2}=25, k_{+}^{2}=100$, and $N=64$.

is deteriorated to second order while the fourth order compact scheme still has fourth order convergence rate until the round-off errors become dominate after $N=512$. It is recommended to use fourth order compact scheme for large wave numbers.

Table 4.6: A comparison of the third and fourth order compact schemes for Example 4.2 with $k_{-}^{2}=1$, $k_{+}^{2}=992.25$. The convergence order for the third and fourth order compact schemes are confirmed

\begin{tabular}{|c|cc|cc|}
\hline & third-order scheme & \multicolumn{2}{|c|}{ fourth-order scheme } \\
\hline$N$ & error & order & error & order \\
\hline 32 & 0.0044 & & $1.7772 \mathrm{e}-5$ & \\
\hline 64 & $9.4124 \mathrm{e}-4$ & 2.2249 & $2.1999 \mathrm{e}-7$ & 6.3360 \\
\hline 128 & $1.1941 \mathrm{e}-4$ & 2.9786 & $1.5525 \mathrm{e}-8$ & 3.8248 \\
\hline 256 & $1.4618 \mathrm{e}-5$ & 3.0301 & $9.2525 \mathrm{e}-10$ & 4.0686 \\
\hline 512 & $1.8018 \mathrm{e}-6$ & 3.0202 & $5.7089 \mathrm{e}-11$ & 4.0186 \\
\hline
\end{tabular}

\section{Conclusions}

In this paper, we have developed the third and fourth order compact finite difference schemes for the Helmholtz equations with piecewise constant wave numbers. The third order scheme is easy to derive and implement. The fourth order scheme matches the fourth order discretization at regular grid points. Quite a few more correction terms are needed compared with the third order one. It is recommended to use the fourth order compact scheme or use fine mesh for large wave numbers.

Acknowledgments. We would like to thank Dr. Semyon Tsynkov for useful discussions. The first author was partially supported by the Key Project of Chinese Ministry of Education 
Table 4.7: A comparison of the third and fourth order compact schemes for Example 4.2 with $k_{-}^{2}=1$, $k_{+}^{2}=90000$. The convergence order for the third order compact scheme was deteriorated to second order due to large wave number while the convergence order for the fourth order one has not been affected.

\begin{tabular}{|c|cc|cc|}
\hline & \multicolumn{3}{|c|}{ the third-order scheme } & \multicolumn{2}{|c|}{ the fourth-order scheme } \\
\hline$N$ & error & order & error & order \\
\hline 16 & 0.0121 & & $1.8688 \mathrm{e}-5$ & \\
\hline 32 & 0.0031 & 1.9647 & $1.1669 \mathrm{e}-6$ & 4.0014 \\
\hline 64 & $8.0227 \mathrm{e}-4$ & 1.9501 & $7.3041 \mathrm{e}-8$ & 3.9978 \\
\hline 128 & $2.0845 \mathrm{e}-4$ & 1.9444 & $4.5590 \mathrm{e}-9$ & 4.0019 \\
\hline 256 & $6.1355 \mathrm{e}-5$ & 1.7644 & $2.8382 \mathrm{e}-10$ & 4.0057 \\
\hline 512 & $2.8563 \mathrm{e}-5$ & 1.1030 & $2.9029 \mathrm{e}-11$ & 3.2894 \\
\hline
\end{tabular}

Grant No. 209134. The first author would like to thank Dr. Zhilin Li and North Carolina State University for the hospitality during the author's visit. The second author was partially supported by the US ARO grants 56349MA-MA, and 550694-MA, and the AFSOR grant FA9550-09-1-0520. The third author was partially supported by Hong Kong Baptist University grant FRG/08-09/II-35.

\section{References}

[1] S. K. Lele. Compact Finite Difference Schemes with Spectral-like Resolution. J. Comput. Phys., 103 (1992), pp. 16-42.

[2] I. Singer and E. Turkel. High-order finite difference methods for the Helmholtz equation. Comput. Methods. Appl. Mech. Engrg., 163 (1998), pp. 343-358.

[3] I. Harari and E. Turkel. Accurate finite difference methods for time-harmonic wave propagation. J. Comput. Phys., 119 (1995), pp. 252-270.

[4] Y. Fu. Compact Fourth-order Finite Difference Schemes for Helmholtz Equation with High Wave numbers. J. Comput. Math., 26 (2008), pp. 98-111.

[5] B. Gustafsson and P. Wahlund. Time compact difference methods for wave propagation in discontinuous media. SIAM J. Sci. Comput., 26 (2004), pp. 272-293.

[6] B. Gustafsson and E. Mossberg. Time compact high order difference methods for wave propagation. SIAM J. Sci. Comput., 26 (2004), pp. 259-271.

[7] G. Baruch, G. Fibich, S. Tsynkov and E. Turkel. Fourth Order Schemes for Time-Harmonic Wave Equations with Discontinuous Coefficients. Commun. Comput. Phys., 5 (2009), pp. 442-455.

[8] M. M. Gupta. A fourth-order poisson solver. J. Comput. Phys., 55 (1984), pp. 166-172.

[9] M. M. Gupta, R. M. Manohar and J. H. Stephenson. A single cell high order scheme for the convection-diffusion equation with variable coefficients. Int. J. Numer. Meth. Fluids, 4 (1984), pp. 641-651.

[10] J. Liu and J. M. Jin. A special high-order finite element method for scattering by deep cavity. IEEE Tran. Antennas Propag., 48 (2000), pp. 694-703.

[11] L. Collatz. The Numerical Treatment of Differential Equations. Springer-Verlag, Berlin, New York, 1960. 
[12] F. Ihlenburg. Finite element analysis of acoustic scattering, vol. 132 of Applied Mathematical Sciences. Springer-Verlag, New York, 1998.

[13] F. Ihlenburg and I. Babuska. Finite element solution of the Helmholtz equation with high wave number Part I: The h-version of FEM. Comput. Math. Appl., 30 (1995), pp. 9-37.

[14] F. Ihlenburg and I. Babuska. Finite element solution of the Helmholtz equation with high wave number Part II: The h-p version of the FEM. SIAM J. Numer. Anal., 34 (1997), pp. 315-358.

[15] G. Baruch, G. Fibich and S. Tsynkov. High-order numerical method for the nonlinear Helmholtz equation with material discontinuities. J. Comput. Phys., 227 (2007), pp. 820-850.

[16] R. D. Ciskowski and C. A. Brebbia. Boundary element methods in Acoustics. Elsevier, London, 1991.

[17] J. Shen and L. Wang. Spectral approximation of the Helmholtz equation with high wave numbers. SIAM J. Numer. Anal., 43 (2006), pp. 623-644.

[18] Q. Fang, D. P. Nicholls and J. Shen. A stable, high-order method for three-dimensional, boundedobstacle, acoustic scattering. J. Comput. Phys., 224 (2007), pp. 1145-1169.

[19] K. Ito, Z. Qiao and J. Toivanen. A domain decomposition solver for acoustic scattering by elastic objects in layered media. J. Comput. Phys., 227 (2008), pp. 8685-8698.

[20] K. Ito and Z. Qiao. A high order compact MAC finite difference scheme for the Stokes equations: Augmented variable approach. J. Comput. Phys., 227 (2008), pp. 8177-8190.

[21] K. Ito and Z. Qiao. A high order finite difference scheme for the Stokes equations. AMS Contem. Math., 466 (2008), pp. 35-51.

[22] R. J. LeVeque and Z. Li. The immersed interface method for elliptic equations with discontinuous coefficients and singular sources. SIAM J. Numer. Anal., 31 (1994), pp. 1019-1044.

[23] Z. Li and K. Ito. The Immersed Interface Method - Numerical Solutions of PDEs Involving Interfaces and Irregular Domains. SIAM Frontier Series in Applied mathematics, FR33, 2006.

[24] R. J. Mackinnon and R. W. Johnson. Differential-equation-based representation of truncation errors for accurate numerical simulation. Int. J. Numer. Meth. Fluids, 13 (1991), pp. 739-757.

[25] G. Bao and W. Sun. A Fast Algorithm for the Electromagnetic Scattering from a Large Cavity. SIAM J. Sci. Comput., 27 (2005), pp. 553-574.

[26] Y. Wang, K. Du and W. Sun. A Second-Order Method for the Electromagnetic Scattering from a Large Cavity. Numer. Math. Theor. Meth. Appl., 1 (2008), pp. 357-382. 